\title{
Improved Dynamic Algorithms for Longest Increasing Subsequence*
}

\author{
Tomasz Kociumaka ${ }^{\dagger}$ \\ kociumaka@berkeley.edu \\ University of California, Berkeley \\ Berkeley, California, USA
}

\author{
Saeed Seddighin $¥$ \\ saeedreza.seddighin@gmail.com \\ Toyota Technological Institute at Chicago \\ Chicago, Illinois, USA
}

\begin{abstract}
We study dynamic algorithms for the longest increasing subsequence (LIS) problem. A dynamic LIS algorithm maintains a sequence subject to operations of the following form arriving one by one: insert an element, delete an element, or substitute an element for another. After each update, the algorithm must report the length of the longest increasing subsequence of the current sequence.

Our main contribution is the first exact dynamic LIS algorithm with sublinear update time. More precisely, we present a randomized algorithm that performs each operation in time $\widetilde{O}\left(n^{4 / 5}\right)$ and, after each update, reports the answer to the LIS problem correctly with high probability. We use several novel techniques and observations for this algorithm that may find applications in future work.

In the second part of the paper, we study approximate dynamic LIS algorithms, which are allowed to underestimate the solution size within a bounded multiplicative factor. In this setting, we give a deterministic $(1-o(1))$-approximation algorithm with update time $O\left(n^{o(1)}\right)$. This result improves upon the previous work of Mitzenmacher and Seddighin (STOC'20) that provides an $\Omega\left(\epsilon^{O(1 / \epsilon)}\right)$ approximation algorithm with update time $\widetilde{O}\left(n^{\epsilon}\right)$ for any $\epsilon>0$.
\end{abstract}

\section{CCS CONCEPTS}

- Theory of computation $\rightarrow$ Dynamic programming.

\section{KEYWORDS}

Longest Increasing Subsequence, dynamic algorithms

ACM Reference Format:

Tomasz Kociumaka and Saeed Seddighin. 2021. Improved Dynamic Algorithms for Longest Increasing Subsequence. In Proceedings of the 53rd Annual ACM SIGACT Symposium on Theory of Computing (STOC '21), Fune 21-25, 2021, Virtual, Italy. ACM, New York, NY, USA, 14 pages. https: //doi.org/10.1145/3406325.3451026

\footnotetext{
"The full version of the paper is available at https://arxiv.org/pdf/2011.10874.pdf [20] ${ }^{\dagger}$ Tomasz Kociumaka is partially supported by NSF 1652303, 1909046, and HDR TRIPODS 1934846 grants, and an Alfred P. Sloan Fellowship.

${ }^{\ddagger}$ Saeed Seddighin is partially funded by an unrestricted gift from Google and a Research Award from Adobe.
}
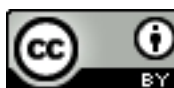

This work is licensed under a Creative Commons Attribution International 4.0 License.

STOC '21, June 21-25, 2021, Virtual, Italy

(c) 2021 Copyright held by the owner/author(s).

ACM ISBN 978-1-4503-8053-9/21/06.

https://doi.org/10.1145/3406325.3451026

\section{INTRODUCTION}

Longest increasing subsequence (LIS) is a very old and classic problem in computer science. In this problem, a sequence $a=$ $\left\langle a_{1}, a_{2}, \ldots, a_{n}\right\rangle$ of size $n$ is given as input and the LIS of the sequence is defined as the largest subset of the elements whose values are strictly increasing in the order of their indices. LIS can also be thought of as a special case of the longest common subsequence (LCS) problem where the two inputs are permutations. Both problems date back to the 1950s and have been subject to a plethora of research $[1,9-13,16,26]$ especially in recent years [17, 22, 23, 27].

In this work, we focus on exact and approximation algorithms in the dynamic setting, where at each step, the sequence can be updated by inserting, deleting, or substituting an element. The goal is to maintain the size of the longest increasing subsequence. Many problems have been studied in dynamic settings; see e.g. [2$4,8,15,18,21,24,25]$. In general, in a dynamic setting, the goal is to develop an algorithm which updates the solution efficiently given incremental changes to the input. In the context of graph algorithms [2-4, 21, 24, 25], such changes are usually modeled by edge insertion or deletion. For string problems, changes are typically modeled with character insertion, deletion, and substitution [6-8], as we consider here.

Our work is closely related to two previous works on dynamic LIS. (In what follows, when we refer to a solution, we typically refer to the size of the LIS, but also the corresponding increasing subsequence can be found in time proportional to its size.) Mitzenmacher and Seddighin [22] give an $\Omega\left(\epsilon^{O(1 / \epsilon)}\right)$-approximation algorithm for dynamic LIS whose update time is bounded by $\widetilde{O}\left(n^{\epsilon}\right)$ for any constant $\epsilon>0$. They also present a $(1+\epsilon)$-approximation algorithm for the dynamic variant of distance to monotonicity, which is the dual of LIS. Their solution for distance to monotonicity requires polylogarithmic update time. Chen, Chu, and Pinkser [8] also study dynamic LIS and give an exact solution whose update time depends on the size of the solution. More precisely, if we denote the solution size by opt, then their algorithm requires update time $O\left(\right.$ opt $\left.\log \frac{n}{\mathrm{opt}}\right)$. Notice that opt can be as large as $\Theta(n)$ and therefore their update time is $\Theta(n)$ in the worst case.

We provide the first exact algorithm for LIS with sublinear update time regardless of the solution size. Specifically, we give a randomized algorithm that processes each update in $O\left(n^{4 / 5} \log ^{3} n\right)$ time $^{1}$ and, after each operation, reports the correct value of the size of the longest increasing subsequence with high probability (at least $\left.1-n^{-10}\right)$. In addition to this, we also present a $(1-o(1))$ approximation algorithm for dynamic LIS that updates the solution

${ }^{1}$ In the full version of this paper [20], we improve the update time to $O\left(n^{2 / 3} \log ^{4} n\right)$. 
Table 1: The results of this paper along with previous work on dynamic LIS. Here, opt denotes the size of the longest increasing subsequence. In the algorithm of Theorem 4.4, if we denote the approximation factor by $1-\epsilon$, the update time will be bounded by $O\left((\log n / \epsilon)^{O\left((\log n)^{2 / 3} / \epsilon\right)}\right)$.

\begin{tabular}{|l|c|c|}
\hline Approximation factor & Update time & Reference \\
\hline $1-\epsilon$ & $\widetilde{O}(\sqrt{n})$ & {$[22]$} \\
\hline$\Omega\left(\epsilon^{O(1 / \epsilon)}\right)$ & $\widetilde{O}\left(n^{\epsilon}\right)$ & {$[22]$} \\
\hline exact & $O\left(\right.$ opt $\left.\log \frac{n}{\text { opt }}\right)$ & {$[8]$} \\
\hline exact & $O\left(n^{4 / 5} \log ^{3} n\right)$ & Theorem 3.2 \\
\hline $1-o(1)$ & $O\left(n^{o(1)}\right)$ & Theorem 4.4 \\
\hline
\end{tabular}

in time $O\left(n^{o(1)}\right)$. Our method substantially advances the techniques of [22] and significantly improves their main results.

\subsection{Parallel and Independent Work}

Parallel to and independent of this work, Gawrychowski and Janczewski [14] presented a different $(1-o(1))$-approximation algorithm for dynamic LIS that updates the solution in subpolynomial time. While the approximation factors of both algorithms are $1-o(1)$, their algorithm is faster: it achieves update time $O\left(\epsilon^{-5} \log ^{11} n\right)$ while maintaining a $(1-\epsilon)$-approximation of LIS. To derive a solution, both approximation algorithms generalize the dynamic LIS problem; our algorithm is able to answer more general queries, though. We also remark that [14] does not claim any progress towards obtaining an exact sublinear-time solution for dynamic LIS.

\subsection{Related Work}

In addition to previous dynamic algorithms for LIS [8, 22], this problem has received significant attention in other areas such as property testing $[1,9-11]$, streaming $[13,16]$, and massively parallel computation (MPC) [19], as well as in the standard algorithmic setting [12, 23, 26-28].

The classic patience sorting solution for LIS uses dynamic programming and binary search to solve LIS exactly in time $O(n \log n)$. Matching lower bounds $(\Omega(n \log n))$ are known for comparisonbased algorithms [12] and solutions based on algebraic decision trees [26]. For approximation algorithms, for any $\epsilon>0$, a multiplicative $\Omega\left(n^{-\epsilon}\right)$-approximate solution can be determined in truly sublinear time ${ }^{2}$ via random sampling ${ }^{3}$. Surprisingly, not much is known that improves upon this algorithm generally, although when $n / \operatorname{LIS}(a)$ is subpolynomial in $n$, we can obtain better approximation guarantees for LIS [23, 27, 28].

From a complexity point of view, unconditional lower bounds apply to sublinear-time algorithms for LIS. For instance, any algorithm that obtains a $(1 / f(n))$-approximate solution for LIS has to make at least $n /(f(n)+1)$ value queries ${ }^{4}$ to the elements of $a$ to distinguish the case that $a$ is decreasing from the case that $a$

\footnotetext{
${ }^{2}$ Truly sublinear stands for $O\left(n^{1-\Omega(1)}\right)$.

${ }^{3}$ For an $\Omega\left(n^{-\epsilon}\right)$-approximation algorithm, one can sample $O\left(n^{1-\epsilon}\right)$ elements from the sequence and report the LIS of those samples.

${ }^{4} \mathrm{~A}$ value query provides an $i$ as input and asks for the value of $a_{i}$.
}

has an increasing subsequence of length at least $f(n)+1$. Thus, a subpolynomial-factor approximation algorithm for LIS in truly sublinear time is not possible in general. In contrast, positive results are given in previous work for a special case in which the solution size is at least $\lambda n$ for large enough $0<\lambda \leq 1$. (The known query complexity lower bounds do not apply to this setting.) Saks and Seshadhri [28] present a $(1-\epsilon)$-approximation algorithm for LIS in this case. The runtime of their algorithm is sublinear as long as $\lambda>\log \log n / \log n$. Moreover, Rubinstein, Seddighin, Song, and Sun [27] give an $\Omega\left(\lambda^{3}\right)$-approximation algorithm for this case in time $\widetilde{O}\left(\sqrt{n} / \lambda^{7}\right)$. Very recently, Mitzenmacher and Seddighin [23] improved the approximation factor to $\Omega\left(\lambda^{\epsilon}\right)$ for any constant $\epsilon>0$ while keeping the runtime truly sublinear.

From a technical standpoint, our approximation algorithm is related to the recent results of $[22,23]$ that approximate LIS in the dynamic and static settings, respectively. Both these works use the grid packing technique for LIS to design their algorithms. We generalize this notion and prove that the generalized grid packing gives improved dynamic algorithms for LIS.

\subsection{Preliminaries}

LIS is defined on a sequence of numbers. We assume for simplicity that all of the numbers are distinct and positive integers. In this problem, the goal is to find the length of the largest subsequence of elements such that their values increase according to their indices. We denote the size of the sequence by $n$ and use $a_{1}, \ldots, a_{n}$ to denote the sequence elements. We also give an alternative definition for the problem which represents the input as $n$ points on the $2 \mathrm{D}$ plane. In this representation, we have $n$ points on the plane with distinct coordinates. Similarly, we assume for simplicity that all the coordinates are positive integers. For any subset of points, its LIS is defined as the largest number of points such that if we sort them based on their $x$ or $y$ coordinates, we obtain the same ordering.

We adapt the setting of [22] for the dynamic LIS problem. Initially, the input sequence is empty $(n=0)$. At each step, an element can either be inserted at an arbitrary position of the sequence or removed from an arbitrary position of the sequence. (Element substitution can be implemented with the previous two operations, so we consider only insertions and deletions.)

We now define the operations more formally. Each insertion is of the form "insert $(i, x)$ ", where $i$ is an integer between 1 and $n+1$. The index $i$ specifies the position of element $x$. After this operation, all the elements whose previous index was at least $i$ will be shifted to the right. Similarly, an operation "delete $(i)$ " removes the $i$ th element. Likewise, all the elements whose previous index was at least $i+1$ will be shifted to the left. As discussed in previous work [22], one can use a balanced tree data structure that provides access to any element of the sequence in time $O(\log n)$. Thus, in the analysis of our time bounds for dynamic LIS, we consider an additional multiplicative $O(\log n)$ overhead for using this data structure and assume that random access is provided to any element.

To simplify the explanations, we often use the notation $\widetilde{O}$ that hides factors polylogarithmic in $n$. When other parameters such as $\epsilon$ or $\kappa$ are involved, we may use $\widetilde{O}_{\epsilon}$ or $\widetilde{O}_{\kappa}$ notations that also hide factors that only depend on $\epsilon$ or $\kappa$. Similarly, $\widetilde{O}_{\epsilon, \kappa}$ hides all the factors that depend on $\epsilon, \kappa$, or (at most polynomially) on $\log n$. 


\section{OUR RESULTS AND TECHNIQUES}

In this section, we present our results and techniques. Our main contribution is a dynamic algorithm for LIS that maintains an exact solution with update time $\widetilde{O}\left(n^{4 / 5}\right)$. We explain the high-level ideas behind this algorithm in Section 2.1. We then proceed by bringing the ideas behind our $(1-o(1))$-approximation algorithm in Section 2.2. For both our dynamic algorithms, we use the notion of block-based algorithms [22] to simplify the exposition. This enables us to include preprocessing steps which may violate the worst-case update times. Yet, a block-based algorithm can be turned into a dynamic algorithm whose worst-case update time is asymptotically equal to the block-based algorithm's amortized update time. More precisely, a block-based algorithm starts with an sequence $a$ of size $n$. It is allowed to preprocess the sequence in time $f(n)$. After the preprocessing step, the algorithm is required to execute $g(n)$ operations, each in worst-case time $h(n)$. After $g(n)$ operations, the block-based algorithm terminates. It follows from [22] that such an algorithm can be used to design a dynamic algorithm for LIS with worst-case update time $O(f(n) / g(n)+h(n))$.

\subsection{An Exact Algorithm with Sublinear Update Time}

Our main result is an exact dynamic algorithm for LIS with sublinear update time. For this algorithm, we use several combinatorial techniques which we explain in the following. The first idea is a randomized coloring argument which lets us decompose the problem into smaller subproblems. Recall that the algorithm of Chen, $\mathrm{Chu}$, and Pinsker [8] provides the exact LIS value with update time $O$ (opt $\log \frac{n}{\mathrm{opt}}$ ), where opt is the solution size. Thus, as long as opt is sublinear in $n$, their algorithm updates the solution in sublinear time. Intuitively, this signals that the real difficulty of the problem is for the case where the LIS is very large, namely, of size $\widetilde{\Omega}(n)$.

We define $b_{j}$ as the size of the longest increasing subsequence ending at element $a_{j}$ and divide the sequence into disjoint layers. More precisely, let each layer $L_{i}=\left\{a_{j} \mid b_{j}=i\right\}$ be the set of elements whose corresponding value $b_{j}$ is equal to $i$. Indeed, the number of distinct layers is equal to the size of the solution and, therefore, when the solution size is large, we expect that the layers are small on average. As an example, if the LIS of the sequence is of size $\Omega(n)$, then we expect the average size of the layers to be $O(1)$ The following observation enables us to decompose the LIS problem into smaller subproblems that can be updated independently: Let opt be the number of layers for a sequence. If, for some integer $t$, we color $t$ layers of the sequence uniformly at random and perform opt/ (10t) arbitrary operations on the sequence, with probability at least $1 / 2$, there exists a longest increasing subsequence in the new sequence that has exactly $t$ colored elements.

A formal proof for the above claim is given in Section 3. Since the element values are decreasing in each layer of the original sequence, no layer can contribute more than one element to any increasing subsequence. Moreover, since the solution size for the original sequence is opt, after opt/(10t) operations, the size of the LIS changes by at most an additive opt/(10t) term. Therefore, if we fix a longest increasing subsequence in the new sequence (after all the operations are performed) and denote its size by opt ${ }^{\prime}$, at least opt - opt/(5t) layers of the original sequence contribute to opt ${ }^{\prime}$.
Since we color $t$ layers uniformly at random, this proves that, with probability at least $1 / 2$, all of the colored layers contribute to opt'

Let us go back to our previous discussion. In a block-based algorithm, we can in time $O(n \log n)$ construct the layers and sample $t$ layers uniformly at random. For the next $g(n)=$ opt $/(10 t)$ operations, we can be sure that, with probability at least $1 / 2$, we have a longest increasing subsequence that goes through all sampled layers. For now, we ignore the bad event and assume for simplicity that this property always holds. Thus, we only need to keep partial solutions between consecutive sampled layers. Since our aim is to design a dynamic algorithm for the case that the size of the longest increasing subsequence is large, we expect that the layer sizes are small. Hence, we assume that, for every pair of elements $a_{i}$ and $a_{j}$ that belong to two consecutive sampled layers, we are given the size of the longest increasing subsequence starting from $a_{i}$ and ending at $a_{j}$, and, for every element of the first sampled layer, we have the size of the longest increasing subsequence ending at that element. Similarly, assume that for each element of the last sampled layer, the size of the longest increasing subsequence starting from that element is available. It follows that, based on this information, we can recover the LIS of the whole sequence (ignoring the bad event). In the extreme case that the solution size opt is $\Omega(n)$, we expect the layer sizes to be $O(1)$ on average, which makes the total size of the information to be stored small.

The benefit of the above approach is obvious for the dynamic setting: If we only care about local solutions between consecutive sampled layers, whenever an operation is performed, we only need to update the local solutions. For this purpose, we only consider elements located between the two consecutive sampled layers. We remark that the positions of such elements in the sequence do not necessarily form an interval. On average, the expected number of such elements is $O(n / t)$. We bring an example to clarify the advantage of this approach: Suppose that the LIS of the original sequence is equal to $\Omega(n)$ and the size of each layer is bounded by $O(1)$. If we set $t=\sqrt{n}$ and sample $t$ layers uniformly at random, we expect to have a solution that goes through all the sampled layers with constant probability for up to $\sqrt{n} / 10$ steps. Thus, we set $g(n)=\sqrt{n} / 10$ and assume that our block-based algorithm is only responsible for performing $g(n)$ operations. For simplicity, we ignore the bad event that a solution may not go through all the sampled layers. Since the size of each layer is $O(1)$, every time an operation arrives, we need to update the solution for at most $O(1)$ pairs of elements, and this can be done in time $\widetilde{O}(\sqrt{n})$ since, with high probability, there are at most $\widetilde{O}(\sqrt{n})$ layers between two consecutive sampled layers (each having $O(1)$ elements). Thus, the update time is $\widetilde{O}(\sqrt{n})$ and, once the solutions between consecutive sampled layers are provided, we can find the longest increasing subsequence of the entire sequence in time $\widetilde{O}(\sqrt{n})$. Therefore, with preprocessing time $f(n)=\widetilde{O}(n)$ and $g(n)=\Omega(\sqrt{n})$, we can update the solution in time $h(n)=\widetilde{O}(\sqrt{n})$, which leads to a dynamic algorithm with update time $\widetilde{O}(\sqrt{n})$.

Roughly speaking, if we only aim to obtain a dynamic algorithm with sublinear update time, the assumption that the solution size is $\widetilde{\Omega}(n)$ does not overly simplify the problem since otherwise we can use the algorithm of Chen, Chu, and Pinsker [8] whose update time is sublinear in that case. The probability $1 / 2$ that a solution 


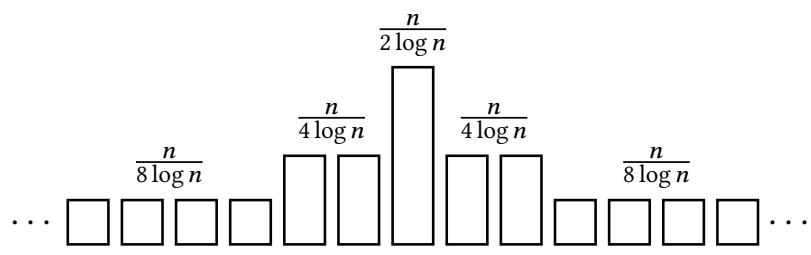

Figure 1: The sizes of the layers in an example are shown in the figure. The middle layer has the largest size and the sizes decrease exponentially as the layers move to the sides.

may fail to go through all the sampled layers (failure probability) can be conveniently reduced to $1-n^{-\Omega(1)}$ via standard techniques at the price of an $O(\log n)$ multiplicative overhead to the runtimes. However, one of the assumptions above does, in fact, oversimplify the problem and may give rise to fundamental issues in general. Even if we assume the solution size is $\widetilde{\Omega}(n)$, we can only guarantee that the size of the layers is small on average and not in the worst case. To illustrate this issue, consider the example given in Fig. 1.

The example of Fig. 1 shows why the above idea alone does not provide a sublinear-time algorithm. Although the size of the LIS is $\widetilde{\Omega}(n)$ and thus the average layer size is $\widetilde{O}(1)$, the two sampled layers that sandwich the middle layer do not yield an easier subproblem. Either the size of one of the sampled layers is large or the number of layers included between them is large. More precisely, the size of the larger sampled layer multiplied by the number of layers between them is $\widetilde{\Omega}(n)$. Therefore, regardless of whether we naively use the patience sorting algorithm to update the local solutions or we use the more sophisticated algorithm of Chen, Chu, and Pinsker [8], the time required to update the local solutions for such sampled layers is $\widetilde{\Omega}(n)$.

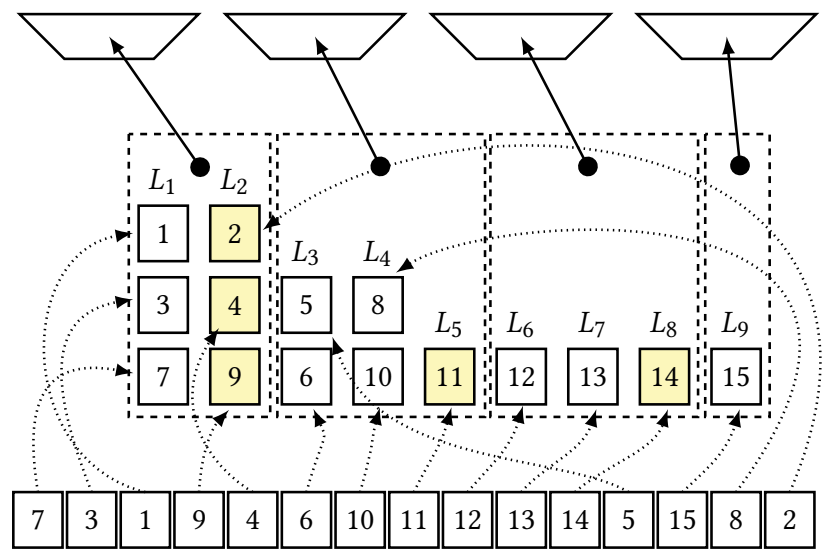

Figure 2: This example shows how the baskets are made from the sampled layers. Sampled layers are colored yellow.

To resolve this issue, we devise a heavy-light decomposition technique to deal with different subproblems. In our algorithm, we make a basket out of consecutive layers that end with a sampled layer; see Fig. 2. Thus, we refer to each of the sampled layers as a boundary layer. For simplicity, we omit some of the details of our algorithm here and only state the overall ideas. (For instance, in our algorithm we do not sample the layers completely at random to make the boundary layers.) Each basket then becomes one subproblem. We define a basket to be light if the total number of elements included in it as well as the size of its boundary layer are both small. In other words, for each of the light baskets, we can store and update the local solutions in sublinear time. A basket is heavy if either its boundary size is large or it contains a large number of elements. In the example of Fig. 1, the basket that contains the middle layer is a heavy basket. For such baskets, we do not store local solutions but, instead, we maintain global information: for each element of a heavy basket, we store the size of the longest increasing subsequence of the entire sequence ending that element.

Because the solutions for heavy baskets are not local, once an operation arrives, after updating the local solution for the corresponding basket (if it is light), we need to propagate the effect of the change. That is, if the local solution for a light basket is affected by a modification, we need to update our solution for all the heavy baskets. To keep the update time sublinear, we make one more observation: It is possible to update the solution for a heavy basket in time proportional to the number of layers included in it. For this purpose, we use the ideas of the work by Chen, Chu, and Pinsker [8], who utilize balanced search trees to obtain a dynamic solution for LIS with update time proportional to the solution size. In the interest of space, here we omit several details of our algorithm; we prove in Section 3 that the combination of these ideas gives us an exact dynamic algorithm for LIS with update time $\widetilde{O}\left(n^{4 / 5}\right)$.

THEOREM 3.2 (RESTATED INFORMALLY). There exists a randomized algorithm for dynamic LIS that has update time $\widetilde{O}\left(n^{4 / 5}\right)$ and, at each step, reports the correct LIS value with probability at least $1-n^{-10}$.

One technical difficulty that arises in the algorithm of Theorem 3.2 is modifying the global information stored within heavy baskets. Although we prove that, with a desirable probability, the longest increasing subsequence of the whole sequence contains an element from each of the sampled layers, this does not hold for all increasing subsequences. Thus, it is likely that, for some element $a_{j}$ in a heavy basket, our algorithm maintains an incorrect value for the longest increasing subsequence ending at $a_{j}$. We discuss this in Section 3 and explain how to overcome the issue.

\subsection{A (1-o(1))-Approximation Algorithm with $O\left(n^{o(1)}\right)$ Update Time}

Our $(1-o(1))$-approximation algorithm for dynamic LIS is based on the notion of grid packing introduced by Mitzenmacher and Seddighin [22]. However, as we discuss later in this section, the original grid packing technique inevitably leads to a constant-factor loss in the approximation ratio. We address this issue by introducing an extended variant of grid packing, which is the basis of our (1 $o$ (1))-approximation algorithm. We explain this in Section 2.2.1. The generalization is natural and inspired by previous work on longest increasing subsequence [19]. However, the more novel and technically challenging component of our algorithm is the application of extended grid packing to dynamic LIS. Since previous applications of grid packing are based on a bound that cannot be guaranteed for a $(1-o(1))$-approximate solution of extended grid 
packing, we design a completely different approach for applying extended grid packing to dynamic LIS. We elaborate more on this in Section 2.2.3. In what follows, we denote the approximation factor of our algorithm by $1-\epsilon$, but remark that subconstant $\epsilon$ is allowed.

2.2.1 Background: Grid Packing. Grid packing is related to the notion of window-compatible solutions proposed by Boroujeni, Ehsani, Ghodsi, HajiAghayi, and Seddighin [5] for approximating edit distance within a constant factor. The problem can be thought of as a game between the algorithm and an adversary. In this problem, we have a table of size $m \times m$. Our goal is to introduce a number of segments on the table. Each segment covers a consecutive set of cells either in a row or in a column. A segment $A$ precedes a segment $B$ if every cell of $A$ is strictly higher than every cell of $B$ and also every cell of $A$ is strictly to the right of every cell of $B$. Two segments are non-conflicting if one of them precedes the other one. Otherwise, we call them conflicting; see Fig. 3. The introduced segments can overlap, and there is no restriction on the number of segments or the length of each segment. However, we would like to minimize the maximum number of segments that cover any cell.

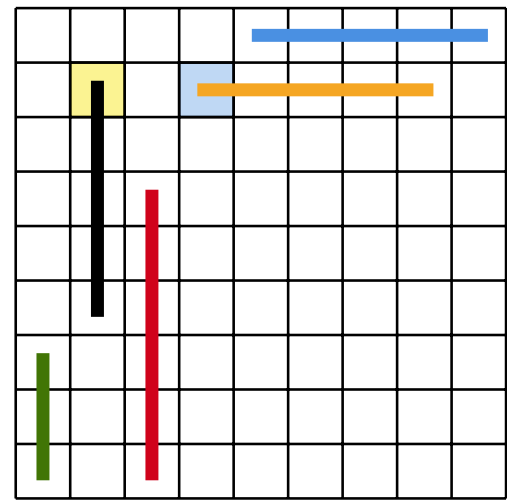

Figure 3: Segments are shown on the grid. The pair (black, orange) is conflicting since the yellow cell (covered by the black segment) is on the same row as the blue cell (covered by the orange segment). The following pairs are non-conflicting: (green, black), (green, orange), (green, blue), (red, orange), (red, blue), (black, blue).

After we choose the segments, an adversary puts a non-negative number on each cell of the table. The score of a subset of cells of the table would be the sum of their values, and the overall score of the table is the maximum score of a path of length $2 m-1$ from the bottom-left corner to the top-right corner. (In such a path, we always either move up or to the right.)

The score of a segment is the sum of the numbers on the cells it covers. We obtain the maximum sum of the scores of a nonconflicting set of segments. The score of the table is an upper bound on the score of any set of non-conflicting segments. We would like to choose segments so that the ratio of the score of the table and our score is bounded by a constant, no matter how the adversary puts the numbers on the table. More precisely, we call a solution $(\alpha, \beta)$-approximate, if at most $\alpha$ segments cover each cell and it guarantees a $1 / \beta$ fraction of the score of the table for us for any assignment of numbers to the table cells.
Mitzenmacher and Seddighin [22] prove the following theorem: For any $m \times m$ table and any $0<\kappa<1$, there exists a grid packing solution with guarantee $\left(O_{\kappa}\left(m^{\kappa} \log m\right), O(1 / \kappa)\right)$. That is, each cell is covered by at most $O_{\kappa}\left(m^{\kappa} \log m\right)$ segments and the ratio of the table's score over our score is bounded by $O(1 / \kappa)$ in the worst case. The proof is constructive and thus gives us the segments explicitly.

2.2.2 Extension: Grid Packing with Multisegments. The general framework of grid packing remains the same for our extension: The problem can be thought of as a game played on an $m \times m$ table against an adversary and the goal is to introduce some multisegments (a generalization of segments explained below) so that after the adversary puts their numbers on the table cells, the score we obtain is comparable to the score of the table. However, extended grid packing differs from grid packing in two ways: First, we introduce a new notion that we call a multisegment and we allow the use of multisegments instead of segments. Second, we do not enforce any bound on the number of multisegments that cover any single cell. That is, our only objective is to maximize the ratio of our score and the score of the table. Without the bound, algorithmically utilizing extended grid packing for LIS becomes more challenging as previous solutions require a cap on the maximum number of segments covering each cell. Nevertheless, we show in Section 4.2 how to apply extended grid packing in absence of this bound.

We bring an example in Section 4 to prove that by just using the segments in the grid packing problem, there is no way to obtain more than a $2 / 3$ fraction of the table's score, even if there is no bound on the number of segments that cover any cell. This example motivates our generalization, which we discuss in the following: For a horizontal (vertical) segment, we define its first cell as its leftmost (resp. bottommost) cell and its last cell as its rightmost (resp. topmost) cell. A $\Delta$-multisegment is defined as a union of $\Delta$ segments $s_{1}, s_{2}, \ldots, s_{\Delta}$ where, for each $1 \leq i \leq \Delta-1$, the last cell of segment $s_{i}$ coincides with the first cell of segment $s_{i+1}$. (By definition, 1-multisegments are the same as segments.) We say that a multisegment covers a cell if any of its segments covers that cell. Moreover, two multisegments $S_{1}$ and $S_{2}$ are non-conflicting if, for each segment $x$ of $S_{1}$ and each segment $y$ of $S_{2}$, the segments $x$ and $y$ are non-conflicting; see Fig. 4. To avoid confusion, we use uppercase letters for multisegments and lowercase letters for segments. Based on this definition, for any $1 \leq i<\Delta$, an $i$-multisegment is also a $\Delta$-multisegment (we may add $\Delta-i$ single cell segments to an $i$-multisegment to make it compatible with the definition of $\Delta$ multisegment without any change in its wording).

We define an extended version of the grid packing problem as a game between the algorithm and an adversary. Similar to grid packing, we first introduce a number of multisegments, and then the adversary puts nonnegative numbers on the cells of the table. The score of the table is defined as the largest sum of values the adversary can collect from the cells by moving from the bottom-left corner to the top-right corner of the table. Our score is the largest total sum of values in cells covered by any set of non-conflicting multisegments.

As we show in Lemma 4.1, if we consider all possible $\Delta$-multisegments in our solution, our score is always at least a $\frac{\Delta-1}{\Delta}$ fraction of the table's score. Notice that by introducing all such multisegments, a cell may be covered by $m^{\Theta(\Delta)}$ multisegments. 


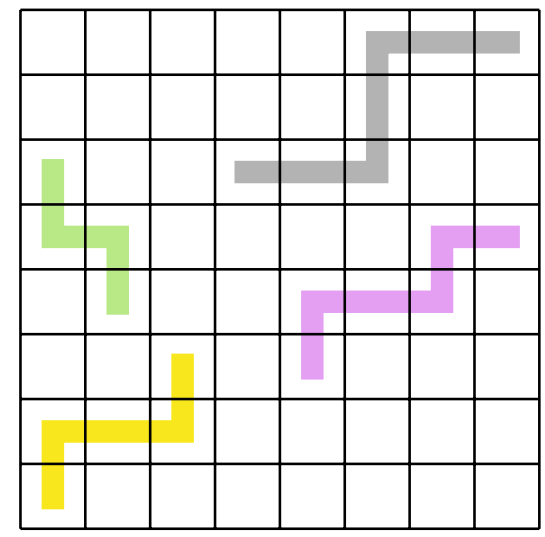

Figure 4: All polylines except for the green one are valid multisegments. Yellow and gray multisegments are non-conflicting, while the remaining multisegment pairs are conflicting.

Lemma 4.1. Let $\Delta$ be a positive integer. If we introduce all $\Delta$ multisegments in the extended grid packing problem, our score will be least a $\frac{\Delta-1}{\Delta}$ fraction of the score of the table regardless of the values of the table cells.

Lemma 4.1 alone does not suffice to improve the dynamic LIS algorithm of Mitzenmacher and Seddighin [22] since the algorithm relies on a bound on the number of segments that cover each cell. We remedy this issue by presenting a more clever algorithm that does not require this bound.

2.2.3 Application of Extended Grid Packing. We refer the reader to previous work [22, 23] for discussions on how to use grid packing for approximating LIS. Roughly speaking, they consider the pointbased representation of the problem and construct an $m \times m$ grid whose rows and columns evenly divide the points. Next, they use the grid packing technique and, for each of the selected segments, they construct a partial solution that maintains an approximation to the LIS of the points covered by that segment. Thus, every time a change is made, their algorithm has to update the solution for all segments that cover the modified point. Therefore, previous techniques require a bound on the number of segments that cover each cell of the grid to make sure the update time is sublinear. In order to obtain a score arbitrarily close to the score of the table, we need to include a lot of multisegments in our solution for extended grid packing, many of which cover the same cells of the table. This renders the previous approach incompatible with the new construction. To address this issue, we introduce a new method that allows using all the $\Delta$-multisegments (for a specific value $\Delta$ ).

At a high-level, the advantage of our new algorithm over the previous technique is that we adaptively decide which multisegments to use in the construction of a global solution. Previous applications are non-adaptive in this sense: They consider all segments of the grid packing solution and, for each segment, they maintain a partial solution for the points covered by that segment. Our solution for extended grid packing uses $m^{O(\Delta)}$ multisegments (for a $(1-\epsilon)$ approximate solution, we require $\Delta=\Omega(1 / \epsilon)$ ) which is too many to even list. Thus, we need to determine which multisegments may potentially contribute to our overall solution before combining the partial solutions. We make such decisions adaptively as we query the subproblems in order to verify if a multisegment can be used in our solution. Thus, as we modify the sequence, the multisegments that may contribute to the global solution are subject to change.

In order to apply extended grid packing, we generalize the dynamic LIS problem. Instead of asking for the size of the longest increasing subsequence after each operation, we define a query to our algorithm in the following way: given a rectangle whose sides are parallel to the axis lines, the algorithm should estimate the size of the longest increasing subsequence of the points in the rectangle. This obviously generalizes the LIS problem since if the rectangle contains all of the points, then the answer is the LIS of the entire sequence. This generalization has two benefits: (i) Instead of defining each subproblem as the points covered by a single segment (as Mitzenmacher and Seddighin [22] do in previous work), we can define each subproblem as the points covered by a row or a column. When the LIS of the points covered by a segment is desired, we can simply query the corresponding part of the subproblem which is covered by the segment. (This does not hold for multisegments in general, but we show how to approximate the LIS of a multisegment in Section 4.) This way, every operation changes at most two subproblems (one row and one column), and thus there is no need to have a bound on the number of segments that cover a point. (ii) The second and more important benefit of this approach is that we can distinguish between the query time and update time. In previous work, there is no distinction between query time and update time since we only look for the LIS of the whole sequence. Therefore, after each operation, the only question that we ask is for the LIS of the entire sequence. With our generalization, we make multiple queries after each update, and therefore answering a query requires a different runtime. One of the key points of our algorithm is that our query time is much smaller than our update time, and this allows us to recursively run multiple queries in each of the subproblems without incurring too much cost in the running time.

Generalized queries, of course, make the problem substantially more complicated. Even in the static setting (when there are no operations to be performed on the sequence), answering this type of queries is not easy. If we only seek the LIS of the entire sequence, patience sorting can solve the problem in nearly linear time. However, if we are allowed to preprocess the sequence and then have to handle queries for the LIS of rectangles, the problem becomes more challenging. The authors are not aware of any linear-time (or even quadratic-time) preprocessing algorithm supporting exact queries in polylogarithmic time. As another application of extended grid packing, we show in Section 4 how to answer the queries in polylogarithmic time with nearly linear-time preprocessing by losing a $1-\epsilon$ factor in the approximation ratio.

A key ingredient of our algorithm is a discretization technique that significantly improves the running time. Roughly speaking, the number of $\Delta$-multisegments grows as $m^{\Theta(\Delta)}$ for an $m \times m$ grid as we increase $\Delta$. Obviously, we cannot afford to consider all such multisegments in our solution. Thus, we need to adaptively decide which multisegments to use in a solution for LIS. To this end, we use a discretization technique that narrows down the search space from all $m^{\Theta(\Delta)}$ multisegments to an $m^{O(1)}\left(\log _{1+\epsilon} n\right)^{O(\Delta)}$-sized subset 
at the expense of losing a $1-\epsilon$ factor in the approximation. This method is technically involved, but enables us to estimate the solution of a query in polylogarithmic time. We explain the details of our algorithm in Section 4.

THEOREM 4.4 (RESTATED INFORMALLY). There exists an algorithm for dynamic LIS that approximates the solution within a $1-o(1)$ multiplicative factor and updates the sequence in $O\left(n^{o(1)}\right)$ time.

\section{EXACT ALGORITHM FOR DYNAMIC LIS}

In this section, we present an exact dynamic algorithm for LIS with sublinear update time. Our method is based on a heavy-light decomposition of regions of the sequence combined with combinatorial analysis of increasing subsequences.

We use the notion of block-based algorithms [22] to simplify the explanation. This lets us include preprocessing steps which may violate the worst-case update times. Yet, a block-based algorithm can be turned into a dynamic algorithm whose worst-case update time is equal to the block-based algorithm's amortized update time. More precisely, a block-based algorithm starts with a sequence $a$ of size $n$. It is allowed to preprocess the sequence in time $f(n)$. After the preprocessing step, the algorithm is required to execute $g(n)$ operations, each in worst-case time $h(n)$. After $g(n)$ operations, the block-based algorithm terminates. As proved in [22], such an algorithm can be transformed into a dynamic algorithm for LIS with worst-case update time $O(f(n) / g(n)+h(n))$.

We construct a block-based algorithm in the following way: In the preprocessing step, we spend time $O(n \log n)$ and compute the size of the longest increasing subsequence that ends at any element $a_{j}$. Let $b_{j}$ denote this value for element $a_{j}$, and let $L_{i}=$ $\left\{a_{j} \mid b_{j}=i\right\}$ be the set of elements whose corresponding solution has size $i$. We refer to each $L_{i}$ as a layer. In the preprocessing step, we construct baskets, each of which consists of the elements of several consecutive layers. Keep in mind that the elements of a basket are not necessarily consecutive in terms of their position in the sequence. However, we maintain as an invariant that every increasing subsequence visits the baskets in the increasing order.

In order to construct the baskets, we define a parameter $w$ that we set later. All baskets, except for the first and the last basket, contain $w$ consecutive layers. We define the boundary of a basket as the last layer included in that basket. We make the baskets in a way that the total size of the boundaries is bounded by $O(n / w)$. Let us be more precise about this. Since each element of the sequence is included in exactly one of the layers, then we have $\sum_{i}\left|L_{i}\right|=n$. Therefore, if we choose an integer value $r$ in range [0,w-1] uniformly at random, then

$$
\mathbb{E}\left[\sum_{i \bmod w=r}\left|L_{i}\right|\right]=\frac{n}{w} .
$$

This implies that $\sum_{i \bmod w}=r\left|L_{i}\right| \leq 2 n / w$ holds for at least $\lceil w / 2\rceil$ choices of $r$. Let $R$ be the set of all such choices for $r$. In our algorithm, we choose a value $r$ from $R$ uniformly at random and set $L_{r}, L_{w+r}, \ldots$ to be the boundaries. If $r \neq 0$, the first basket contains all layers $L_{1}, L_{2}, \ldots, L_{r}$; otherwise, it contains the first $w$ layers. The second basket contains the next $w$ layers and the same holds for the rest of the baskets. If the last basket contains fewer than $w$ layers, we create an additional dummy layer that only contains a

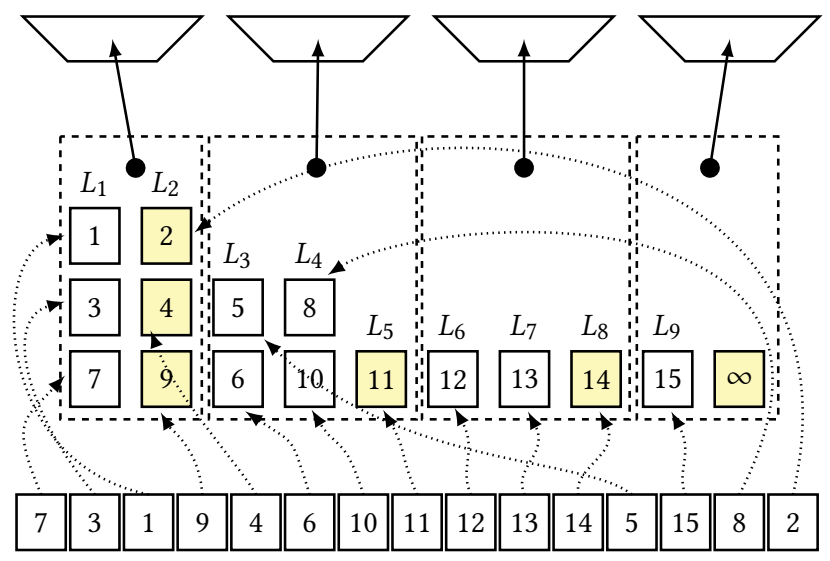

Figure 5: In this example, we have $w=3$ and $r=2$. The boundary layers are colored yellow. The last basket contains a dummy layer with a single element with value $\infty$. We assume that this element is located to the right of all elements.

dummy element with position $n+1$ and value $\infty$ and put this in the last basket as the boundary; see Fig. 5. Otherwise, we create a new basket that has a single element with position $n+1$ and value infinity. This element is also the boundary of the last basket. We denote by $d_{i}$ the size of basket $i$ (the number of elements included in it) and by $e_{i}$ the size of the boundary of basket $i$.

We emphasize that the layers, baskets, and their boundaries are essentially kept intact for the lifetime of the algorithm (which is at most $g(n)$ updates), even though the optimal increasing subsequences may change. The only exception is that each element inserted to the sequence $a$ is inserted to one of the existing baskets, and each element removed from the sequence $a$ is removed from its basket. The newly inserted elements are never included in a boundary, and the algorithm declares a failure following any attempt to remove a boundary element (which technically means that the algorithm ignores subsequent updates and keeps reporting 0 as the LIS length). Since the updates that the algorithm encounters are independent of the random choice of $r$, any single update leads to a failure with probability at most $1 /|R|<2 / w$, which makes the overall failure probability limited to $2 g(n) / w$.

Instead of maintaining the LIS, our algorithm actually maximizes the length of an increasing subsequence that includes one element from each boundary (except for the last one consisting of $a_{n+1}=\infty$ ). Before we describe how this is achieved, let us argue why this is meaningful.

Lемма 3.1. Let $U$ be an arbitrary longest increasing subsequence after at most $g(n)$ updates. Then, with probability at least $1-4 g(n) / w$, the subsequence $U$ contains an element form each boundary layer (except for the last one consisting of $a_{n+1}=\infty$ ).

Proof. Note that $|U| \geq$ opt $-g(n)$, where opt denotes the LIS length during the initialization. Moreover, at most $g(n)$ elements of $U$ may have been added after the initialization, which means that at least $|U|-2 g(n)$ elements were present in the original sequence. Any increasing subsequence contains at most one element from each layer, so $U$ may miss up to $2 g(n)$ out of the opt layers constructed 
during initialization. Any layer $L_{i}$ is a boundary layer if $i \bmod$ $w=r$, which happens with probability $1 /|R| \leq 2 / w$, and thus the probability that $U$ misses a boundary layer is at most $4 g(n) / w$.

Since the failure probability is $2 g(n) / w$, Lemma 3.1 implies that each answer reported by the algorithm is correct with probability at least $1-6 g(n) / w$. We set $g(n)=w / 12$ to make sure that this is at least $1 / 2$. (To further boost the success probability, our final solution maintains $O(\log n)$ instances of the algorithm presented here; see the proof of Theorem 3.2 for details.)

Let us proceed with the details of our algorithm. It follows from our construction that each basket contains at most $w$ consecutive layers (the equality holds with the exception of the first and the last baskets). Moreover, the total size of the boundaries is bounded by $2 n / w+1$ (the additional +1 term is due to the dummy layer). Let $w \leq s \leq n$ be a parameter that we set later. We use $s$ as a threshold on the size of the baskets that we call light. We categorize the baskets based on their number elements and boundary size:

- We call a basket light if its size is bounded by $s$, the size of its boundary (the number of elements in its last layer) is bounded by $s / w$, and the size of the boundary of its previous basket (if any) is bounded by $s / w$.

- If a basket is not light, we call it heavy. That is, a heavy basket is of size more than $s$, its boundary size is more than $s / w$, or its previous basket has a boundary of size more than $s / w$.

Since the total number of elements is bounded by $n$ and the total size of all boundaries is bounded by $2 n / w+1$, then the number of heavy baskets is bounded by $5 n / s+1$.

Throughout our algorithm, we maintain a local data structure for each light basket $i$ that stores the following information: If $i=1$, then, for each element $a_{y}$ of basket 1 , we store the size of the longest increasing subsequence that ends at $a_{y}$. If $i>1$, then, for each boundary element $a_{x}$ of basket $i-1$ and each element $a_{y}$ of basket $i$, we store the size of the longest increasing subsequence that starts at $a_{x}$ and ends at $a_{y}$. Except for the initial element $a_{x}$, such a subsequence may only contain elements from basket $i$.

In the preprocessing step, we initialize all these data structures. We can initialize the local data structure for basket $i>1$ in time $O\left(e_{i-1} d_{i} \log n\right)$ by running patience sorting for each boundary element of basket $i-1$ separately. The size of each light basket is bounded by $s$ and the total boundary size (across all baskets) is at most $2 n / w+1$, so the total time for initializing all light baskets $i>1$ is $O(n s \log n / w)$. Initializing basket 1 (if it is light) costs $O\left(d_{1} \log n\right)=O(s \log n)$ time, and this does not change the overall preprocessing time asymptotically.

We also keep some information for each heavy basket, but that information is not local. In other words, it depends on the elements of the previous baskets as well. (In contrast, the data structure that we keep for each light basket is completely local.) Let $a_{j}$ be an element in basket $i$. We call an increasing subsequence ending at $a_{j}$ basket-compatible if it includes one element from the boundary of each basket $1,2, \ldots, i-1$. For every element $a_{j}$, we denote by $b_{j}^{\prime}$ the size of the longest basket-compatible increasing subsequence that ends at $a_{j}$. Initially, we have $b_{j}=b_{j}^{\prime}$ for all elements but the values $b_{j}^{\prime}$ may diverge from $b_{j}$ as we update to the sequence.
For each heavy basket, we partition its elements into disjoint sets. Each set contains elements $a_{j}$ whose values $b_{j}^{\prime}$ are equal. Initially, each of these sets is one of the layers included in the basket. As we modify the sequence, the values $b_{j}^{\prime}$ change and thus these sets may no longer coincide with the layers. Nevertheless, there is no extra preprocessing cost for heavy baskets since these sets are initially equal to the layers.

When an operation arrives, we first locate the basket to which it relates. We only consider insertions and deletions as substitutions can be simulated by the first two operations. A deletion corresponds to the basket that contains the removed element. An insertion corresponds to the basket with the lowest index such that none of its boundary elements is both to the left of the added element and has a smaller value. In other words, when a new element is added, we find the basket with the lowest index such that none of its boundary elements can be used to update the solution for the added element. (This way, we maintain the invariant that every increasing subsequence visits the baskets in the increasing order.) If the corresponding basket is light, then we update the local data structure in time $O(s(s / w) \log n)=O\left(s^{2} \log n / w\right)$ (recall that the size of each light basket is bounded by $s$ and the previous basket has its boundary size bounded by $s / w$ ). If the operation corresponds to a heavy basket, we do not make any local changes.

After local changes, we compute global solutions for all baskets in the following way: starting from basket 1 , for each boundary element $a_{j}$, we compute $b_{j}^{\prime}$. After we do this for basket 1 , we move on to basket 2 and proceed to the last basket. The computed value for the dummy element minus 1 is the value that we output.

Recall that $e_{i}$ denotes the size of the boundary of basket $i$. For a light basket, we can use the local data structure and update the solution for its boundary elements in time $O\left(e_{i-1} e_{i}\right)$. Since the total size of the boundaries is bounded by $2 n / w+1$ (i.e., $\sum e_{i} \leq 2 n / w+1$ ) and since $e_{i} \leq s / w$ if basket $i$ is light, then the total runtime for light baskets is bounded by $O\left(n s / w^{2}\right)$. For each heavy basket, we use the algorithm of Chen, $\mathrm{Chu}$, and Pinsker [8] to update the solution in time $O(w \log n)$ (more details are given in the proof of Theorem 3.2). Since there are at most $5 n / s+1$ heavy baskets, the total update time for heavy baskets is $O(n w \log n / s)$. By setting $w=n^{2 / 5}$ and $s=n^{3 / 5}$, we obtain a block-based algorithm with $f(n)=$ $O(n s \log n / w)=O\left(n^{6 / 5} \log n\right), g(n)=w / 12=n^{2 / 5} / 12$, and $h(n)=$ $O\left(s^{2} \log n / w+n s / w^{2}+n w \log n / s\right)=O\left(n^{4 / 5} \log n\right)$. This leads to a dynamic algorithm for LIS with update time $O\left(n^{4 / 5} \log n\right)$ that, after each update, reports the solution correctly with probability at least $1 / 2$. By adding an additional $\log n$ multiplicative factor to the update time, we can reduce the error probability to $n^{-10}$. There is one more $O(\log n)$ factor in the update time due to the data structure that we use for accessing the elements of the sequence.

THEOREM 3.2. There exists a randomized algorithm for the $d y$ namic LIS problem that has update time $O\left(n^{4 / 5} \log ^{3} n\right)$ and maintains the value of LIS correctly with probability at least $1-n^{-10}$.

Proof. As discussed earlier, we design a block-based algorithm with preprocessing time $f(n)=O\left(n^{6 / 5} \log n\right)$ which is responsible for updating the solution for up to $g(n)=n^{2 / 5} / 12$ operations and updates the solution in worst-case time $h(n)=O\left(n^{4 / 5} \log n\right)$. To this end, we set $s=n^{3 / 5}, w=n^{2 / 5}$, and divide the elements into 
different baskets. By the discussion following Lemma 3.1, after each update, we report the correct LIS value with probability at least $1 / 2$.

To bring the failure probability down to $n^{-10}$, we repeat the same procedure $20 \log n$ times. That is, we choose $20 \log n$ different values $r$ from $R$ and each time we make the baskets according to different boundaries. After each update, we output the maximum answer that we obtain from all the $20 \log n$ algorithms. Also, since we use a balanced tree to access the elements of the sequence, another $O(\log n)$ factor is also involved in the runtime which makes the overall update time $O\left(n^{4 / 5} \log ^{3} n\right)$.

Another thing to note here is the algorithm that we use for the heavy baskets. Chen, Chu, and Pinsker [8] showed how to maintain LIS with update time $O($ opt $\log (n /$ opt $))$, where opt is the size of the solution. In their algorithm, they divide the elements into subsets $L_{1}^{\prime}, L_{2}^{\prime}, \ldots, L_{\mathrm{opt}}^{\prime}$ such that the longest increasing subsequence ending at each element of $L_{i}^{\prime}$ has size $i$. We use $L^{\prime}$ to denote these subsets since we use $L_{i}$ for the layers of our algorithm. The difference between $L_{i}$ and $L_{i}^{\prime}$ is that our algorithm keeps the sets $L_{i}$ intact while their algorithm updates the sets $L_{i}^{\prime}$ as the sequence changes.

The algorithm of Chen, Chu, and Pinsker [8] is based on the following observation: when an operation is performed to the sequence, each element may only move between consecutive subsets and, moreover, the elements that move form an interval. In other words, if three elements $a_{i}>a_{j}>a_{k}$ belong to a subset, it is impossible for elements $a_{i}$ and $a_{k}$ to move to another subset while $a_{j}$ remains in the same subset after the update. They show that, based on these two properties, after each operation, we can update the solution in time $O$ (opt $\log (n /$ opt $))$.

We use the same idea for heavy baskets. More precisely, for each heavy basket, we divide the elements into subsets $L_{\alpha}^{\prime}, L_{\alpha+1}^{\prime}, \ldots$, where $L_{i}^{\prime}$ consists of elements $a_{j}$ for which the size $b_{j}^{\prime}$ of the longest basket-compatible increasing subsequence ending at $a_{j}$ is equal to $i$. We emphasize that these subsets are different from the layers $L_{1}, L_{2}, \ldots$ since the layers do not change as we perform operations to the sequence. Clearly, after each operation, elements can only move between consecutive subsets of $L_{i}^{\prime}$ in a heavy basket. Moreover, if in a subset $L_{i}^{\prime}$ of a heavy basket some elements move, the moving elements form an interval. To prove this, we show a reduction from the longest basket-compatible increasing subsequence to the longest increasing subsequence. We know that this property holds for the longest increasing subsequence. Now, from our sequence $a$, we make another sequence $a^{\prime}$ which is the same as $a$ except that we copy each boundary element $n$ times and put the copies next to each other. (To make the values distinct, we add $i \epsilon$ to the $i$ th copy of each boundary element.) It follows that, for each element $a_{i}^{\prime}$ of the new sequence, if we find the longest increasing subsequence ending at $a_{i}^{\prime}$ and remove the copied elements from the solution, we obtain the longest basket-compatible increasing subsequence that ends at its corresponding element in sequence $a$. Since performing a single operation in $a^{\prime}$ preserves the interval property of moving elements, this means that the same also holds for $a$ when we are concerned with basket-compatible increasing subsequences.

Initially, the values $b_{j}^{\prime}$ across elements in any single basket span $w$ consecutive integers. Each operation changes the value $b_{j}^{\prime}$ by at most one, so at any time the values $b_{j}^{\prime}$ across elements in any single basket span at most $w+2 g(n) \leq 14 w / 12$ consecutive integers.
Therefore, the cost of updating any heavy basket is $O(w \log n)$, which yields $O(n w \log n / s)$ time across all the heavy baskets.

Thus, as discussed above, we obtain a block-based algorithm with $f(n)=O(n s \log n / w)=O\left(n^{6 / 5} \log n\right), g(n)=w / 12=n^{2 / 5} / 12$, and $h(n)=O\left(s^{2} \log n / w+n s / w^{2}+n w \log n / s\right)=O\left(n^{4 / 5} \log n\right)$. This leads to a dynamic algorithm for LIS with update time $O\left(n^{4 / 5} \log n\right)$ that, after each update, reports the solution correctly with probability at least $1 / 2$. Two multiplicative $O(\log n)$ factors are added to the update time due to the data structure we use to access the elements of the sequence and $20 \log n$ different instances that we run in parallel to reduce the error probability down to $n^{-10}$.

\section{IMPROVED APPROXIMATION ALGORITHMS FOR LIS}

In this section, we present applications of extended grid packing. We begin by stating our key lemma for extended grid packing in Section 4.1. Then, in Section 4.2, we illustrate the extended grid packing technique using a static toy problem. This makes it clear how to use new technique for LIS approximation. We then provide a more detailed discussion as to why extended grid packing leads to a dynamic algorithm for LIS. While, at a high-level, both our dynamic and static algorithms make use of extended grid packing in a similar way, the dynamic algorithm requires additional considerations to ensure the update time remains bounded in the worst case.

\subsection{Extended Grid Packing}

As explained earlier, grid packing is a tool for approximating LIS. For completeness, we first state the definitions. In this problem, we have a table of size $m \times m$. Our goal is to introduce a number of segments on the table. Each segment covers a consecutive set of cells either in a row or in a column. A segment $A$ precedes a segment $B$ if every cell of $A$ is strictly higher than every cell of $B$ and also every cell of $A$ is strictly to the right of every cell of $B$. Two segments are non-conflicting if one of them precedes the other one. Otherwise, we call them conflicting. The introduced segments can overlap, and there is no restriction on the number of segments or the length of each segment. However, we would like to minimize the maximum number of segments that cover any cell.

After we choose the segments, an adversary puts a non-negative number on each cell of the table. The score of a subset of cells of the table would be the sum of their values, and the overall score of the table is the maximum score of a path of length $2 m-1$ from the bottom-left corner to the top-right corner. (In such a path, we always either move up or to the right.)

The score of a segment is the sum of the numbers on the cells it covers. We obtain the maximum sum of the scores of a nonconflicting set of segments. The score of the table is an upper bound on the score of any set of non-conflicting segments. We would like to choose segments so that the ratio of the score of the table and our score is bounded by a constant, no matter how the adversary puts the numbers on the table. More precisely, we call a solution $(\alpha, \beta)$-approximate if at most $\alpha$ segments cover each cell and it guarantees a $1 / \beta$ fraction of the score of the table for us for any assignment of numbers to the table cells.

Mitzenmacher and Seddighin [22] prove the following theorem: For any $m \times m$ table and any $0<\kappa<1$, there exists a grid packing 
solution with guarantee $\left(O_{\kappa}\left(m^{\kappa} \log m\right), O(1 / \kappa)\right)$. That is, each cell is covered by at most $O_{\kappa}\left(m^{\kappa} \log m\right)$ segments and the ratio of the table's score over our score is bounded by $O(1 / \kappa)$ in the worst case.

The general framework of grid packing remains the same for our extension: The problem can be thought of as a game played on an $m \times m$ table against an adversary, and the goal is to introduce some multisegments (a generalization of segments explained below) such that, after the adversary puts their numbers on the table cells, the score we obtain is comparable to table's score. However, extended grid packing differs with grid packing in two ways: First, we introduce a new notion that we call a multisegment and we allow the use of multisegments instead of segments. Second, we do not enforce any bound on the number of multisegments that cover each cell. That is, our only objective is to maximize the ratio of our score over the score of the table. Without the bound, utilizing extended grid packing for LIS becomes harder because previous solutions require a cap on the maximum number of segments covering each cell. However, in Section 4.2, we present a more clever application of extended grid packing that does not depend on this bound.

Before we introduce multisegments, let us give an example to illustrate why segments fall short of our purpose which is obtaining a $1-\epsilon$ fraction of the table's score. For an $m \times m$ table, there are $m\left(\begin{array}{c}m \\ 2\end{array}\right)+m^{2}$ distinct horizontal segments and $m\left(\begin{array}{c}m \\ 2\end{array}\right)+m^{2}$ distinct vertical segments that amount to $2 m\left(\begin{array}{c}m \\ 2\end{array}\right)+m^{2}$ segments in total (there are $m^{2}$ single-cell segments that can be regarded as both vertical and horizontal). Fig. 6 gives an example proving that we cannot obtain a score exceeding $2 / 3$ of the score of the table. In this example, even if we introduce all possible $2 m\left(\begin{array}{c}m \\ 2\end{array}\right)+m^{2}$ segments, from every three consecutive cells with value 1 , no more than two can be covered by non-conflicting segments. Thus, we cannot obtain

\begin{tabular}{|c|c|c|c|c|c|c|c|}
\hline 0 & 0 & 0 & 0 & 0 & 0 & 0 & 1 \\
\hline 0 & 0 & 0 & 0 & 0 & 0 & $\begin{array}{l}r \\
1\end{array}$ & -1 \\
\hline 0 & 0 & 0 & 0 & 0 & $\left|\begin{array}{cc}- & \\
1 & \end{array}\right|$ & 1 & 0 \\
\hline 0 & 0 & 0 & 0 & 1 & $\left.\mid \begin{array}{lll}1 & & 1 \\
1 & 1 & 1\end{array}\right)$ & 0 & 0 \\
\hline 0 & 0 & 0 & $r_{-}^{-}$ & $\begin{array}{r}-1 \\
-1\end{array}$ & 0 & 0 & 0 \\
\hline 0 & 0 & $\begin{array}{c}- \\
1\end{array}$ & 1 & 0 & 0 & 0 & 0 \\
\hline 0 & 1 & $\mid \begin{array}{lll} & 1 & 1 \\
& \end{array}$ & 0 & 0 & 0 & 0 & 0 \\
\hline $\begin{array}{l}r_{1}^{-} \\
1\end{array}$ & $\begin{array}{c}-\overline{1} \\
11\end{array}$ & 0 & 0 & 0 & 0 & 0 & 0 \\
\hline
\end{tabular}

Figure 6: Only the cells of one path from the bottom-left to the top-right have value 1 and the value of the remaining cells is $\mathbf{0}$. We can obtain a score of $\mathbf{1 0}$ by using the green segments, but we miss 5 cells with value 1 (colored in orange). Any other non-conflicting set of segments also misses at least 5 cells with value 1 . more than $2 / 3$ of the score of the table even if there is no restriction on the maximum number of segments covering each cell.

The example of Fig. 6 highlights the fact that even if all possible segments can be used in a solution, there is no hope to obtain a $1-\epsilon$ fraction of the score of the table. This is the main motivation behind the definition of multisegments. As we show later, multisegments enable us to obtain a score arbitrarily close to the score of the table.

For a horizontal (vertical) segment, we define its first cell as its leftmost (resp. bottommost) cell and its last cell as its rightmost (resp. topmost) cell. A $\Delta$-multisegment is defined as a union of $\Delta$ segments $s_{1}, s_{2}, \ldots, s_{\Delta}$ where, for each $1 \leq i \leq \Delta-1$, the last cell of segment $s_{i}$ coincides with the first cell of segment $s_{i+1}$. (By definition, 1-multisegments are the same as segments.) We say that a multisegment covers a cell if any of its segments covers that cell. Moreover, two multisegments $S_{1}$ and $S_{2}$ are non-conflicting if, for each segment $x$ of $S_{1}$ and each segment $y$ of $S_{2}$, the segments $x$ and $y$ are non-conflicting. To avoid confusion, we use uppercase letters for multisegments and lowercase letters for segments. Based on this definition, for any $1 \leq i<\Delta$, an $i$-multisegment is also a $\Delta$-multisegment (we may add $\Delta-i$ single cell segments to an $i$-multisegment to make it compatible with the definition of $\Delta$ multisegment without any change in its wording).

We define an extended version of the grid packing problem as a game between the algorithm and an adversary. Similar to grid packing, we first introduce a number of multisegments, and then the adversary puts nonnegative numbers on the cells of the table. The score of the table is defined as the largest sum of values the adversary can collect from the cells by moving from the bottom-left corner to the top-right corner of the table. Our score is the largest total sum of values in cells covered by any set of non-conflicting multisegments.

As shown in Lemma 4.1, if, for a given $\Delta \geq 1$, we consider all possible $\Delta$-multisegments in our solution, our score is always at least a $\frac{\Delta-1}{\Delta}$ fraction of the table's score. Notice that by introducing all such multisegments, a cell may be covered by $m^{\Theta(\Delta)}$ multisegments. In the interest of space, we skip the proof of Lemma 4.1 here, but the reader can find that in the full version of the paper [20].

LEMMA 4.1. Let $\Delta$ be a positive integer. If we introduce all $\Delta$ multisegments in the extended grid packing problem, our score will be least $a \frac{\Delta-1}{\Delta}$ fraction of the score of the table regardless of the values of the table cells.

Lemma 4.1 alone does not suffice to improve the dynamic LIS algorithm of Mitzenmacher and Seddighin [22] since the algorithm relies on a bound on the number of segments that cover each cell. We remedy this issue by presenting, in Section 4.3, a more clever algorithm that does not require this bound.

\subsection{A Data Structure for LIS Queries}

Previous works $[22,23]$ illustrate natural connections between LIS and grid packing. Generally, we represent the input sequence by points on the plane in a way that a point with coordinates $(x, y)$ indicates that the $x$ th element of the sequence has value $y$. Thus, instead of a sequence of length $n$, we have a plane with $n$ points. This enables us to construct a grid, where the rows and the columns evenly divide the points. Assuming that the number the adversary puts on each cell is the contribution of the points inside that cell to 
the LIS of the sequence (which may be different from the LIS of all the points included in that cell), the score of the table (which we try to be competitive against in extended grid packing) is equal to the size of the optimal solution. Moreover, the notion of non-conflicting (multi)segments gives us a way to construct a global solution by combining local partial solutions. In other words, if we associate a partial solution to each (multi)segment that only incorporates the points covered by this (multi)segment, then, for a set of nonconflicting (multi)segments, the union of partial solutions forms a valid increasing subsequence.

We refer the reader to previous work [22, 23] for discussions on how to use grid packing for approximating LIS. Roughly speaking, they consider the point-based representation of the problem and construct an $m \times m$ grid whose rows and columns evenly divide the points. Next, they use the grid packing technique and, for each of the selected segments, they construct a partial solution that maintains an approximation to the LIS of the points covered by that segment. Thus, every time a change is made, their algorithm has to update the solution for all segments that cover the modified point For this reason, previous techniques require a bound on the number of segments that cover each cell of the grid to make sure the update time is sublinear. In order to obtain a score arbitrarily close to the score of the table, we need to include a lot of multisegments in our solution for extended grid packing, many of which cover the same cells of the table. This renders the previous approach incompatible with the new construction. To address this issue, we introduce a new method that allows using all the $\Delta$-multisegments (for a specific value $\Delta$ ). To illustrate the new technique, we apply extended grid packing to approximate the LIS sizes in a static setting. We later bring a more involved description of the dynamic algorithm.

Assume that we are given $n$ points on the plane with distinct coordinates. We would like to preprocess the points so that, afterwards, we can (approximately) answer the following queries: Given a rectangle parallel to the axis lines, what is the LIS of the points contained in the rectangle? Recall that the LIS of a set of points is equal to the size of the longest list of the points where the $x$ and $y$ coordinates increase as the index of the elements increase in the list. To avoid confusion, we assume that the $x$ and $y$ coordinates of the borders of the rectangles are different from the coordinates of the points. We refer to these queries as LIS queries.

There is a simple solution answering LIS queries in $O(\log n)$ time after $O\left(n^{5} \log n\right)$-time preprocessing. We first sort the $x$ and $y$ coordinates separately and, for every interval of the $x$ coordinates and $y$ coordinates, we compute the LIS of the points within those intervals. Since there are $O\left(n^{2}\right)$ intervals for $x$ coordinates and $O\left(n^{2}\right)$ intervals for $y$ coordinates, in total we compute the LIS of $O\left(n^{4}\right)$ subsets of the points, and thus the overall preprocessing time is $O\left(n^{5} \log n\right)$. After this, whenever we are given a rectangle, we find the $x$ - and $y$-intervals of the points covered by the rectangle and report the solution in time $O(\log n)$. The preprocessing time can be easily improved to $O\left(n^{4} \log n\right)$ and beyond, but, for the sake of simplicity, we do not discuss those improvements here.

Instead, we explain an application of extended grid packing that improves the preprocessing time for LIS queries down to $\widetilde{O}\left(n^{5 / 2}\right)$ while keeping the query time polylogarithmic. This comes at the expense of losing a factor $1-\epsilon$ in the approximation ratio. To this end, we define $\bar{\epsilon}=\epsilon / 2$ and $\Delta=\lceil 1 / \bar{\epsilon}\rceil$. Let $m=n^{5 / 8}$ be the table size in the extended grid packing problem; we construct the table in a way that the rows and columns evenly divide the points (if the number of points is not divisible by $m$, we allow differences of \pm 1 in the number of points covered by each row and column). After constructing the table, we define $2 m$ subproblems, each concerning the points that fall within a row or a column. That is, in each subproblem, we seek to answer LIS queries for rectangles contained either in a row or in a column of the table. Thus, the size of each subproblem is $O\left(n^{3 / 8}\right)$. We use the naive solution with preprocessing time of $O\left(\left(n^{3 / 8}\right)^{5} \log n\right)$ for each subproblem. This amounts to a total preprocessing time of

$$
O\left(2 m\left(\frac{n}{m}\right)^{5} \log n\right)=O\left(\frac{n^{5} \log n}{m^{4}}\right)=O\left(\frac{n^{5} \log n}{\left(n^{5 / 8}\right)^{4}}\right)=O\left(n^{5 / 2} \log n\right) .
$$

Before answering the queries, we run another algorithm to precompute approximate LIS values for some subsets of the points. More precisely, for every interval of rows and every interval of columns of the table, we precompute the LIS of all the points that fall within the corresponding rectangle. In other words, we approximate the LIS for $\left(\left(\begin{array}{c}m \\ 2\end{array}\right)+m\right)^{2}$ rectangles consisting of full table cells.

In our algorithm, we fix the bottom-left corner of the rectangle and use dynamic programming to approximate the solutions for all possible top-right corners. Thus, in what follows, we fix a cell $c$ of the table and explain how we can approximate the value of LIS for all rectangles whose bottom-left corner is $c$ in time $\widetilde{O}_{\epsilon}\left(m^{2}\right)$.

For each cell $c^{\prime}$ which is neither to the left of $c$ nor below $c$, we define $f\left(c^{\prime}\right)$ as the LIS of the points inside the rectangle formed by $c$ and $c^{\prime}$ as opposite corners, and we use $g\left(c^{\prime}\right)$ to denote an approximation of $f\left(c^{\prime}\right)$. We compute the values $g\left(c^{\prime}\right)$ iteratively. Therefore, we start with $c^{\prime}=c$ and move $c^{\prime}$ to the right step by step. When we reach the end of the row, we start with the cell above $c$ and move it to the right until we reach the end of the row, and then we start with the second cell above $c$. We continue this procedure until we find a solution for the top-right corner as the last rectangle.

In the DP, we use extended grid packing to estimate the LIS for each rectangle. The base cases are when $c^{\prime}$ and $c$ are either within the same row or within the same column, in which case we retrieve the exact value $f\left(c^{\prime}\right)$ from one of the subproblems in time $O(\log n)$. Otherwise, we estimate the value of $f\left(c^{\prime}\right)$ based on an approach inspired by extended grid packing on our $m \times m$ table. In our solution for extended grid packing, we introduce all possible $\Delta$-multisegments. Moreover, we assume that the numbers that the adversary puts on the table cells are the contributions of the corresponding cells to the LIS of the elements in the rectangle between $c$ and $c^{\prime}$. By Lemma 4.1, there is a set of non-conflicting $\Delta$-multisegments that gives us a score of at least $\frac{\Delta-1}{\Delta} f\left(c^{\prime}\right)$. Furthermore, the LIS of the points covered by any multisegment is certainly an upper bound on the value of that multisegment. Let $X$ be the set of all cells in the rectangle between $c$ and $c^{\prime}$, except for $c^{\prime}$, and, for any cell $c^{\prime \prime}$, let $Y\left(c^{\prime \prime}\right)$ be the set of all $\Delta$-multisegments that end at $c^{\prime}$ and start at the cell above and to the right of $c^{\prime \prime}$. Moreover, for a multisegment $S$, we define $\operatorname{LIS}(S)$ as the LIS of the points covered by $S$. For now, we introduce the following recursive formula for approximating $f\left(c^{\prime}\right)$ :

$$
g\left(c^{\prime}\right):=\max _{c^{\prime \prime} \in X}\left[g\left(c^{\prime \prime}\right)+\max _{S \in Y\left(c^{\prime \prime}\right)} \operatorname{LIS}(S)\right]
$$


It follows from Lemma 4.1 that, if we define $g\left(c^{\prime}\right)$ as above, then $\frac{\Delta-1}{\Delta} f\left(c^{\prime}\right) \leq g\left(c^{\prime}\right) \leq f\left(c^{\prime}\right)$ always holds. This gives us a formulation to recursively compute the value of $g$ for all cells. However, there are two issues to be resolved: (i) there are many possible cells $c^{\prime \prime}$ and multisegments $S$ that our algorithm needs to loop over, and thus the runtime of the algorithm is not as desired. (ii) For a multisegment $S$, we do not have the value (or even an estimate) of $\operatorname{LIS}(S)$. We show in the following that both issues can be resolved.

We begin by considering the simpler case of $\Delta=1$. Since in this case we are only concerned with segments, for each segment $s$, we can compute LIS $(s)$ by querying one of the subproblems (recall that $s$ either completely fits in a row or in a column of the table). Thus, for each segment $s, \operatorname{LIS}(s)$ is available in time $O(\log n)$. However, we still need to resolve the first issue since there may be up to $O(m)$ different segments that end at $c^{\prime}$. The idea is to reduce the number of possible segments down to $O(\log n / \bar{\epsilon})$ by losing a factor of at most $1-\bar{\epsilon}$ in the approximation. The base cases are trivial as discussed previously, so, for a cell $c^{\prime}$, we begin by initializing $g\left(c^{\prime}\right)$ as the maximum value for the cells below and to the left of $c^{\prime}$. This ensures monotonicity of our approximations. At a high-level, for every value $v \in \mathbb{D}=\left\{1,\lceil 1-\bar{\epsilon}\rceil,\left\lceil(1-\bar{\epsilon})^{2}\right\rceil,\left\lceil(1-\bar{\epsilon})^{3}\right\rceil, \ldots, n\right\}$, we only consider the shortest segments $s$ that end at $c^{\prime}$ and satisfy $\operatorname{LIS}(s) \geq v$. There are at most $O(\log n / \bar{\epsilon})$ such vertical segments and at most $O(\log n / \bar{\epsilon})$ such horizontal segments; each one can be found via a binary search in time $O\left(\log ^{2} n\right)(\operatorname{an} O(\log n)$ overhead for binary search and an $O(\log n)$ overhead for finding the LIS of a potential solution). Thus, if we only consider these segments, the runtime improves to $O\left(\log ^{3} n / \bar{\epsilon}\right)$ for each pair of cells $\left(c, c^{\prime \prime}\right)$, which in total amounts to a runtime of $O\left(m^{4} \log ^{3} n / \bar{\epsilon}\right)$.

The correctness of this procedure is easy. Assume that the optimal segment that gives us the highest value for $g\left(c^{\prime}\right)$ in the original algorithm is a horizontal segment $s_{1}$, but we do not consider $s_{1}$ in the improved algorithm. Let $v$ be the largest value in sequence $\mathbb{D}$ which satisfies $v \leq \operatorname{LIS}\left(s_{1}\right)$. Let the corresponding horizontal segment for value $v$ in our algorithm be $s_{2}$. We define $c_{1}^{\prime \prime}, c_{2}^{\prime \prime}$ as the cells to the left and below the first cell of $s_{1}$ and $s_{2}$, respectively. It follows from our algorithm that $s_{1}$ is at least as long as $s_{2}$, and thus $g\left(c_{2}^{\prime \prime}\right) \geq g\left(c_{1}^{\prime \prime}\right)$ by monotonicity of $g$. Moreover, we have $\operatorname{LIS}\left(s_{2}^{\prime \prime}\right) \geq v \geq(1-\bar{\epsilon}) \operatorname{LIS}\left(s_{1}^{\prime \prime}\right)$ so, in the update process, we only lose an $\bar{\epsilon}$ fraction of the LIS of the last segment. The same analysis works for vertical segments as well. This implies that our estimation for $g$ loses a factor of at most $(1-\bar{\epsilon})$ throughout the DP.

For $\Delta=2$, we first consider all 1-multisegments (segments) as explained above and determine an initial value for $g\left(c^{\prime}\right)$. Then, we proceed with a generalization of the above idea for 2-multisegments. Let $\mathbb{D}=\left\{1,\lceil 1-\bar{\epsilon}\rceil,\left\lceil(1-\bar{\epsilon})^{2}\right\rceil,\left\lceil(1-\bar{\epsilon})^{3}\right\rceil, \ldots, n\right\}$. For any two values $v_{1}, v_{2} \in \mathbb{D}$, we consider the following 2-multisegment: We define a sweeping line which is initially equal to the right edge of cell $c^{\prime}$ We move the sweeping line parallel to that edge to the left, until the LIS of the rectangle which is covered by the first sweeping line is at least $v_{1}$. Let $e$ be the cell that contains the sweeping line. Now, we define another sweeping line which is equal to the portion of the bottom edge of $e$ which is not covered by the first sweeping line. Starting from there, we move the second sweeping line parallel to that edge downward, until the LIS of the corresponding rectangle becomes at least $v_{2}$. We define a 2-multisegment $S$ as the set of all the cells that intersect with either rectangles and estimate $\operatorname{LIS}(S)$ by $v_{1}+v_{2}$. Next, we determine cell $c^{\prime \prime}$ which is to the left and below the first cell of $S$, and we update the value of $g\left(c^{\prime}\right)$ as $g\left(c^{\prime}\right):=$ $\max \left\{g\left(c^{\prime}\right), g\left(c^{\prime \prime}\right)+v_{1}+v_{2}\right\}$. Similarly, we repeat the same procedure starting with vertical and then horizontal rectangles. The total number of 2-multisegments that we investigate via this algorithm is $2|\mathbb{D}|^{2}=O\left(\log ^{2} n / \bar{\epsilon}^{2}\right)$, and finding each 2-multisegments takes time $O\left(\log ^{2} n\right)$. Thus, the running time becomes $O\left(\log ^{4} n / \bar{\epsilon}^{2}\right)$ for each pair of cells $\left(c, c^{\prime}\right)$, and $O\left(m^{4} \log ^{4} n / \bar{\epsilon}^{2}\right)$ in total.

Let us prove that we only lose a factor of at most $1-\bar{\epsilon}$ in the approximation ratio. Similar to the previous discussion, let $S^{\prime}$ be the last multisegment that is used in the original algorithm to update the value of $g\left(c^{\prime}\right)$. If $S^{\prime}$ fits in a row or column (meaning it is a segment), then the proof follows from our previous discussion. Thus, without loss of generality, we assume that $S^{\prime}$ consists of a horizontal segment (on top) and a vertical segment (on the bottom). Let $v_{1}^{\prime}$ be the contribution of the top horizontal part of $S^{\prime}$ to $\operatorname{LIS}\left(S^{\prime}\right)$ and $v_{2}^{\prime}$ be the contribution of the remainder of $S^{\prime}$ to $\operatorname{LIS}\left(S^{\prime}\right)$. We define $v_{1}$ and $v_{2}$ as the largest numbers in the set $\mathbb{D}$ that are bounded by $v_{1}^{\prime}$ and $v_{2}^{\prime}$, respectively. In our algorithm, we consider pair of values $\left(v_{1}, v_{2}\right)$ for constructing a 2-multisegment in the following way: We first make a rectangle via a sweeping line that moves horizontally from $c^{\prime}$ to the left. Then, we do the same thing downward for $v_{2}$.

Let the two rectangles be $R_{1}$ and $R_{2}$. If rectangle $R_{1}$ does not touch the top-left cell of $S^{\prime}$, then $g\left(c^{\prime}\right)$ can be updated via a segment with parameter $v_{1}$. This is because, without the horizontal part of $S^{\prime}$, the LIS of the remainder of $S^{\prime}$ is at least $v_{2}^{\prime}$. Thus, if we define $d^{\prime}$ as the second last cell of the vertical segment of $S^{\prime}$, then $g\left(d^{\prime}\right) \geq g\left(c^{\prime}\right)-v_{1}^{\prime}$. Hence, if we define $d$ to be the cell to the left and below the first cell of $R_{1}$, then $g(d) \geq g\left(d^{\prime}\right) \geq g\left(c^{\prime}\right)-v_{1}^{\prime}$. Thus, we only lose a $1-\bar{\epsilon}$ factor if we update $g\left(c^{\prime}\right)$ using a segment with parameter $v_{1}$.

If $R_{1}$ intersects the top-left cell of $S^{\prime}$, then $R_{2}$ also falls within $S^{\prime}$ and, therefore, the 2-multisegment that our algorithm constructs will be entirely inside $S^{\prime}$. Moreover, the LIS of the 2-multisegment that our algorithm makes is estimated as $v_{1}+v_{2} \geq(1-\bar{\epsilon})\left(v_{1}^{\prime}+v_{2}^{\prime}\right)$. Thus, we only lose a factor $1-\bar{\epsilon}$ in our estimation.

This approach is generalizable to larger $\Delta$. In order to update the value for $g\left(c^{\prime}\right)$, for any tuple of $\Delta$ elements $v_{1}, v_{2}, \ldots, v_{\Delta} \in \mathbb{D}$, we construct a $\Delta$-multisegment by making $\Delta$ rectangles, and we estimate the value of the multisegment by $v_{1}+v_{2}+\cdots+v_{\Delta}$. Next, we update the value of $g\left(c^{\prime}\right)$ from the constructed multisegment. This takes time $O\left(\Delta m^{4} \log ^{\Delta+2} n / \bar{\epsilon}^{\Delta}\right)$ since making each $\Delta$-multisegment costs $O\left(\Delta \log ^{2} n\right)$ time. The analysis of the approximation ratio resembles the argument for 2-multisegments.

After a $O\left(\Delta m^{4} \log ^{\Delta+2} n / \bar{\epsilon}^{\Delta}\right)$-time preprocessing, for every pair of table cells, we have a $(1-\bar{\epsilon}) \frac{\Delta-1}{\Delta} \geq 1-\epsilon$ approximation of the LIS of the points included in the rectangle between the two corners. We show that, using this information, we will be able to answer each query in time $O\left(\log ^{2 \Delta+2} n / \bar{\epsilon}^{2 \Delta+2}\right)$. The idea is similar to what we do above. Again, we consider solutions made by non-conflicting multisegments. The only difference is that we only take into account the points that lie inside the given query rectangle. Moreover, instead of $\Delta$-multisegments, we consider $(\Delta+1)$-multisegments here. Since we already have a desirable estimation for any rectangle that starts from a table cell and ends at another table cell, we only need to fix the bottom-left and top-right multisegment. Using the above 
idea, we construct $O\left(\log ^{\Delta+1} n / \bar{\epsilon}^{\Delta+1}\right)$ different candidate $(\Delta+1)$ multisegments for bottom-left and $O\left(\log ^{\Delta+1} n / \bar{\epsilon}^{\Delta+1}\right)$ different candidate $(\Delta+1)$-multisegments for top-right corner. This amounts to $O\left(\log ^{2 \Delta+2} n / \bar{\epsilon}^{2 \Delta+2}\right)$ combinations, so we obtain an $\frac{\Delta-1}{\Delta}(1-\bar{\epsilon}) \geq$ $1-\epsilon$ approximation of the solution in $O\left(\log ^{2 \Delta+2} n / \bar{\epsilon}^{2 \Delta+2}\right)$ time. Note that once we fix the bottom-left and top-right multisegments, the solution for the area between them is already available.

The reason we use $(\Delta+1)$-multisegments instead of $\Delta$-multisegments is the following: consider the optimal non-conflicting $\Delta$ multisegments that provide the solution for a query. They may not necessarily cover the bottom-left and top-right corners of the queryrectangle. Using $(\Delta+1)$-multisegments, we can modify the optimal non-conflicting $\Delta$-multisegments to cover both corners as well.

THEOREM 4.2. For any $0<\epsilon, \kappa$, LIS queries can be approximated within a factor $1-\epsilon$ with query time $O\left(\left(\log \frac{1}{\kappa} \log \frac{n}{\epsilon}\right) O\left(\frac{1}{\epsilon}\left(\log \frac{1}{\kappa}\right)^{2}\right)\right)$ and preprocessing time $O\left(\left(\log \frac{1}{\kappa} \log \frac{n}{\epsilon}\right) O\left(\left(\frac{1}{\epsilon} \log \frac{1}{\kappa}\right)^{2}\right) n^{1+\kappa}\right)$.

Theorem 4.2 is proved in the full version of the paper [20].

\subsection{A Dynamic Solution for LIS}

We present a dynamic algorithm for LIS that approximates the solution within a $1-\epsilon$ multiplicative factor. This is achieved by using extended grid packing. For the purpose of our technique, we generalize the problem in the following way: In the original dynamic problem, our goal is to maintain an approximation to the size of the longest increasing subsequence. Thus after each operation, one needs to update the solution size and, therefore, we can simply assume that the LIS size is reported after each update. In our generalization, we still consider the same set of operations. However, our algorithm is required to answer a stronger type of queries: Assuming that we describe the sequence as points on the plane (as discussed earlier), for each query, we provide a rectangle with edges parallel to the axis lines, and the algorithm should provide an estimate for the LIS of the points inside the rectangle. This is similar to the LIS queries considered in Section 4.2.

Since, in the previous setting, we only asked for the size of the longest increasing subsequence, one could argue that, after each update, there is just one question to be answered. In our new setting, we may need to answer multiple queries after each update, and thus it makes sense to separate the concepts of queries and updates. That is, we may be able to answer each query faster than the time which our algorithm requires for updating the sequence. Thus, in our setting, we define the update time to be the time our algorithm needs to update the sequence after an operation arrives, and the query time to be the time our algorithm needs to answer a query. We show in the following that we can answer each query faster than the update time, and this is an important part of the analysis.

We show that, for any $0<\epsilon, \kappa$, our algorithm provides a $(1-\epsilon)$ approximation of the solution with update time $\widetilde{O}_{\epsilon, \kappa}\left(n^{\kappa}\right)$ and query time $\widetilde{O}_{\epsilon, \kappa}(1)$. Recall that $\widetilde{O}_{\epsilon, \kappa}$ hides all the factors that depend only on $\epsilon, \kappa$, or (at most polynomially) on $\log n$. An exact dynamic solution for LIS with update time $O\left(n^{5} \log n\right)$ and query time $O(\log n)$ follows from the straightforward algorithm discussed in Section 4.2. In other words, if, after every operation, we compute the solution for all possible rectangles that can be given to the algorithm as a query, then we can answer each query in time $O(\log n)$. Moreover, since there are at most $O\left(n^{4}\right)$ rectangles that cover distinct sets of points, we can update the solution in time $O\left(n^{5} \log n\right)$ whenever an operation arrives. Notice that after answering a query, we do not require to update the solution. To improve the update time, we use the notion of block-based algorithms [22]: if we design a block-based algorithm whose bound over the update time is amortized, we can then turn the block-based algorithm into an equivalent dynamic algorithm with a worst-case bound on its update time.

For a block-based algorithm, we start with an initial sequence of size $n$. Our algorithm is then allowed to preprocess the input in time $f(n)$. After the preprocessing phase, our block-based algorithm is responsible for $g(n)$ operations and all the queries that come prior to the last operation. After $g(n)$ operations, our algorithm terminates. Mitzenmacher and Seddighin [22] prove that if the block-based algorithm performs each operations in time $h(n)$ in the worst case, then it can be transformed into a dynamic algorithm whose worstcase update time is $O(f(n) / g(n)+h(n))$. The approximation factor of the dynamic algorithm is exactly the same as the block-based algorithm and also the query time remains asymptotically the same. Since Mitzenmacher and Seddighin [22] use a slightly different setting in which queries and operations are treated equivalently, we bring a formal proof for our discussion in the full version [20].

To improve upon the naive algorithm, we design a block-based algorithm with preprocessing time $f(n)=O\left(n^{19 / 7} \log n\right), g(n)=$ $n^{3 / 7}$, and $h(n)=\widetilde{O}_{\epsilon}\left(n^{16 / 7}\right)$. In the preprocessing phase, we construct a grid of size $m \times m$, where $m=n^{4 / 7}$. The rows and columns of our grid evenly divide the points, and thus each row or column covers $O\left(n^{3 / 7}\right)$ points. Similar to what we discussed in Section 4.2, we make $2 m$ subproblems for dynamic LIS where each subproblem is concerned with the subset of points that is covered by the corresponding row or the corresponding column of the grid. The construction of the grid only requires sorting the points based on $x$ and $y$ coordinates, and thus we can do it in time $O(n \log n)$. Moreover, each subproblem includes $O\left(n^{3 / 7}\right)$ points, and therefore precomputing the solutions for all rectangles within each subproblem takes time $O\left(n^{15 / 7} \log n\right)$. Since we run this procedure for all $2 m$ subproblems, the total preprocessing time is $O\left(n^{19 / 7} \log n\right)$.

Due to our construction, each time an update arrives, only one row and one column is affected. Also, since the size of each subproblem (the number of points covered by each row or column) is bounded by $O\left(n^{3 / 7}\right)$, then, by precomputing the solution for all possible rectangles, we can update the entries for each subproblem in time $O\left(n^{15 / 7} \log n\right)$. In addition to updating the solution for each subproblem, we also maintain a table of size $m^{2} \times m^{2}$ that keeps an approximation to the value of LIS for each rectangle consisting of full grid cells. As discussed in Section 4.2, using the extended grid packing, we can approximate the solution for each rectangle within a factor $1-\epsilon$ and doing this for all possible rectangles takes time $\widetilde{O}_{\epsilon}\left(\mathrm{m}^{4}\right)$. Therefore, maintaining the table costs $\widetilde{O}_{\epsilon}\left(m^{4}\right)=\widetilde{O}_{\epsilon}\left(n^{16 / 7}\right)$ time per update. Similar to the algorithm of Section 4.2 , each query can be approximated via the precomputed solutions for the rectangles and the solutions for subproblems in time $\widetilde{O}_{\epsilon}(1)$. Since $g(n)=n^{3 / 7}$, we are sure that after $g(n)$ operations, the size of each subproblem remains bounded by $O\left(n^{3 / 7}\right)$, and thus the runtimes do not increase asymptotically as operations 
add new points to the subproblems. Therefore, our block-based algorithm has preprocessing time $f(n)=O\left(n^{19 / 7} \log n\right), g(n)=n^{3 / 7}$, and $h(n)=\widetilde{O}_{\epsilon}\left(n^{16 / 7}\right)$. This leads to a dynamic solution with worstcase update time $\widetilde{O}_{\epsilon}\left(n^{16 / 7}\right)$ and approximation ratio $1-\epsilon$; the query time of our dynamic algorithm is $\widetilde{O}_{\epsilon}(1)$.

Since inserting elements to the sequence or removing elements from the sequence may change the indices of other elements, the points on the plane are subject to moves. Each time an operation arrives, we update the solution for the corresponding subproblems. Let us be more specific about this. Initially, $m-1$ vertical lines divide the array into chunks of size roughly $n / m$. As operations arrive, the elements are shifted to the left or to the right (their indices are updated). Each vertical line can be thought of as a separator between two consecutive elements that is also shifted to the left or to the right when elements are added or removed. Thus, although the vertical lines move, each element which is inserted or deleted lies between two thresholds and corresponds to a unique column of the grid. The corresponding row is uniquely determined by the horizontal lines (those lines remain unchanged). Thus, every element insertion or deletion affects only one cell of the grid which is included in at most two subproblems. Mitzenmacher and Seddighin [22] show that the shifts can be efficiently simulated with an $O(\log n)$ overhead in the runtime of the algorithm. More precisely, they give a data structure that is able to insert and delete elements from the sequence while giving access to any position of the sequence in time $O(\log n)$. We use the same data structure and therefore we incorporate an additional $O(\log n)$ overhead in the runtime of our dynamic algorithms.

In the full version of the paper [20], we bring a recursive application of the above ideas that leads to a solution with update time $\widetilde{O}_{\epsilon, \kappa}\left(n^{\kappa}\right)$ and approximation ratio $1-\epsilon$ for arbitrarily small $0<\epsilon, \kappa$. The query time of our algorithm is $\widetilde{O}_{\epsilon, \kappa}(1)$.

Theorem 4.3. For any $0<\epsilon, \kappa$, there exists a dynamic algorithm for LIS with worst-case update time $O\left((\log n /(\epsilon \kappa))^{O\left(1 /\left(\epsilon \kappa^{2}\right)\right)} n^{\kappa}\right)$, approximation ratio $1-\epsilon$, and query time $O\left((\log n /(\epsilon \kappa))^{O\left(1 /\left(\epsilon \kappa^{2}\right)\right)}\right)$.

By setting $\kappa=1 /(\log n)^{1 / 3}$ in Theorem 4.3, we obtain an algorithm with update time $O\left((\log n / \epsilon)^{O\left((\log n)^{2 / 3} / \epsilon\right)}\right)$. In this case, the query time and update time are equal, so we can use this algorithm for the original dynamic problem wherein the queries and the updates are treated the same way.

Theorem 4.4 (a corollary of Theorem 4.3). For any $0<\epsilon$, there exists a dynamic algorithm for LIS with worst-case update time $O\left((\log n / \epsilon)^{O\left((\log n)^{2 / 3} / \epsilon\right)}\right)$ and approximation ratio $1-\epsilon$.

\section{REFERENCES}

[1] Nir Ailon, Bernard Chazelle, Seshadhri Comandur, and Ding Liu. 2007. Estimating the distance to a monotone function. Random Struct. Algorithms 31, 3 (2007), 371-383. https://doi.org/10.1002/rsa.20167

[2] Sepehr Assadi, Krzysztof Onak, Baruch Schieber, and Shay Solomon. 2018. Fully dynamic maximal independent set with sublinear update time. In STOC 2018 ACM. https://doi.org/10.1145/3188745.3188922

[3] Sepehr Assadi, Krzysztof Onak, Baruch Schieber, and Shay Solomon. 2019. Fully Dynamic Maximal Independent Set with Sublinear in $n$ Update Time. In SODA 2019. SIAM. https://doi.org/10.1137/1.9781611975482.116

[4] Soheil Behnezhad, Mahsa Derakhshan, MohammadTaghi HajiAghayi, Cliff Stein, and Madhu Sudan. 2019. Fully Dynamic Maximal Independent Set with Polylogarithmic Update Time. In FOCS 2019. IEEE. https://doi.org/10.1109/FOCS.2019. 00032
[5] Mahdi Boroujeni, Soheil Ehsani, Mohammad Ghodsi, Mohammad Taghi HajiAghayi, and Saeed Seddighin. 2018. Approximating Edit Distance in Truly Subquadratic Time: Quantum and MapReduce. In SODA 2018. SIAM. https: //doi.org/10.1137/1.9781611975031.76

[6] Panagiotis Charalampopoulos, Paweł Gawrychowski, and Karol Pokorski. 2020. Dynamic Longest Common Substring in Polylogarithmic Time. In ICALP 2020. Schloss Dagstuhl-Leibniz-Zentrum für Informatik. https://doi.org/10.4230/ LIPIcs.ICALP.2020.27

[7] Panagiotis Charalampopoulos, Tomasz Kociumaka, and Shay Mozes. 2020. Dynamic String Alignment. In CPM 2020. Schloss Dagstuhl-Leibniz-Zentrum für Informatik. https://doi.org/10.4230/LIPIcs.CPM.2020.9

[8] Alex Chen, Timothy Chu, and Nathan Pinsker. 2013. The Dynamic Longest Increasing Subsequence Problem. arXiv:1309.7724

[9] Yevgeniy Dodis, Oded Goldreich, Eric Lehman, Sofya Raskhodnikova, Dana Ron, and Alex Samorodnitsky. 1999. Improved Testing Algorithms for Monotonicity. In RANDOM 1999. Springer. https://doi.org/10.1007/978-3-540-48413-4_10

[10] Funda Ergün, Sampath Kannan, Ravi Kumar, Ronitt Rubinfeld, and Mahesh Viswanathan. 2000. Spot-Checkers. 7. Comput. Syst. Sci. 60, 3 (2000), 717-751. https://doi.org/10.1006/jcss.1999.1692

[11] Eldar Fischer. 2004. The art of uninformed decisions: A primer to property testing. In Current Trends in Theoretical Computer Science. World Scientific, 229-263. https://doi.org/10.1142/9789812562494 0014

[12] Michael L. Fredman. 1975. On computing the length of longest increasing subsequences. Discret. Math. 11, 1 (1975), 29-35. https://doi.org/10.1016/0012365X(75)90103-X

[13] Anna Gál and Parikshit Gopalan. 2010. Lower Bounds on Streaming Algorithms for Approximating the Length of the Longest Increasing Subsequence. SIAM $\mathcal{F}$. Comput. 39, 8 (2010), 3463-3479. https://doi.org/10.1137/090770801

[14] Paweł Gawrychowski and Wojciech Janczewski. 2021. Fully Dynamic Approximation of LIS in Polylogarithmic Time. In STOC 2021. ACM. https: //doi.org/10.1145/3406325.3451137 arXiv:2011.09761

[15] Paweł Gawrychowski, Adam Karczmarz, Tomasz Kociumaka, Jakub Łącki, and Piotr Sankowski. 2018. Optimal Dynamic Strings. In SODA 2018. SIAM. https: //doi.org/10.1137/1.9781611975031.99

[16] Parikshit Gopalan, T. S. Jayram, Robert Krauthgamer, and Ravi Kumar. 2007. Estimating the sortedness of a data stream. In SODA 2007. SIAM. http://dl.acm. org/citation.cfm?id=1283383.1283417

[17] MohammadTaghi HajiAghayi, Masoud Seddighin, Saeed Seddighin, and Xiaorui Sun. 2019. Approximating LCS in Linear Time: Beating the $\sqrt{n}$ Barrier. In SODA 2019. SIAM. https://doi.org/10.1137/1.9781611975482.72

[18] Monika Henzinger, Sebastian Krinninger, Danupon Nanongkai, and Thatchaphol Saranurak. 2015. Unifying and Strengthening Hardness for Dynamic Problems via the Online Matrix-Vector Multiplication Conjecture. In STOC 2016. ACM. https://doi.org/10.1145/2746539.2746609

[19] Sungjin Im, Benjamin Moseley, and Xiaorui Sun. 2017. Efficient massively parallel methods for dynamic programming. In STOC 2017. ACM. https://doi.org/10. $1145 / 3055399.3055460$

[20] Tomasz Kociumaka and Saeed Seddighin. 2021. Improved Dynamic Algorithms for Longest Increasing Subsequence. arXiv:2011.10874

[21] Jakub Łącki, Jakub Oćwieja, Marcin Pilipczuk, Piotr Sankowski, and Anna Zych. 2015. The Power of Dynamic Distance Oracles: Efficient Dynamic Algorithms for the Steiner Tree. In STOC 2015. ACM. https://doi.org/10.1145/2746539.2746615

[22] Michael Mitzenmacher and Saeed Seddighin. 2020. Dynamic Algorithms for LIS and Distance to Monotonicity. In STOC 2020. ACM. https://doi.org/10.1145/ 3357713.3384240

[23] Michael Mitzenmacher and Saeed Seddighin. 2021. Improved Sublinear Time Algorithm for Longest Increasing Subsequence. In SODA 2021. SIAM. https: //doi.org/10.1137/1.9781611976465.115

[24] Danupon Nanongkai and Thatchaphol Saranurak. 2017. Dynamic spanning forest with worst-case update time: adaptive, Las Vegas, and $O\left(n^{1 / 2-\epsilon}\right)$-time. In STOC 2017. ACM. https://doi.org/10.1145/3055399.3055447

[25] Danupon Nanongkai, Thatchaphol Saranurak, and Christian Wulff-Nilsen. 2017. Dynamic Minimum Spanning Forest with Subpolynomial Worst-Case Update Time. In FOCS 2017. IEEE. https://doi.org/10.1109/FOCS.2017.92

[26] Prakash Ramanan. 1997. Tight $\Omega(n \log n)$ lower bound for finding a longest increasing subsequence. Int. F. Comput. Math. 65, 3-4 (1997), 161-164. https: //doi.org/10.1080/00207169708804607

[27] Aviad Rubinstein, Saeed Seddighin, Zhao Song, and Xiaorui Sun. 2019. Approximation Algorithms for LCS and LIS with Truly Improved Running Times. In FOCS 2019. IEEE. https://doi.org/10.1109/FOCS.2019.00071

[28] Michael E. Saks and C. Seshadhri. 2017. Estimating the Longest Increasing Sequence in Polylogarithmic Time. SIAM f. Comput. 46, 2 (2017), 774-823. https: //doi.org/10.1137/130942152 